\title{
Modeling of plates with multiple anisotropic layers and residual stress
}

\author{
Engholm, Mathias; Pedersen, Thomas; Thomsen, Erik Vilain
}

Published in:

Sensors and Actuators A: Physical

Link to article, DOI:

10.1016/j.sna.2016.01.054

Publication date:

2016

Document Version

Peer reviewed version

Link back to DTU Orbit

\section{Citation (APA):}

Engholm, M., Pedersen, T., \& Thomsen, E. V. (2016). Modeling of plates with multiple anisotropic layers and residual stress. Sensors and Actuators A: Physical, 240, 70-79. https://doi.org/10.1016/j.sna.2016.01.054

\section{General rights}

Copyright and moral rights for the publications made accessible in the public portal are retained by the authors and/or other copyright owners and it is a condition of accessing publications that users recognise and abide by the legal requirements associated with these rights.

- Users may download and print one copy of any publication from the public portal for the purpose of private study or research.

- You may not further distribute the material or use it for any profit-making activity or commercial gain

- You may freely distribute the URL identifying the publication in the public portal 


\title{
Modeling of Plates with Multiple Anisotropic Layers and Residual Stress
}

\author{
Mathias Engholm, Thomas Pedersen, Erik Vilain Thomsen \\ Department of Micro- and Nanotechnology, DTU Nanotech, Technical University of Denmark, Building 345E, DK-2800 Lyngby, Denmark
}

\begin{abstract}
Usually the analytical approach for modeling of plates uses the single layer plate equation to obtain the deflection and does not take anisotropy and residual stress into account. Based on the stress-strain relation of each layer and balancing stress resultants and bending moments, a general multilayered anisotropic plate equation is developed for plates with an arbitrary number of layers. The exact deflection profile is calculated for a circular clamped plate of anisotropic materials with residual bi-axial stress. From the deflection shape the critical stress for buckling is calculated and by using the Rayleigh-Ritz method the natural frequency is estimated. Using the Galerkin method, an approximate deflection shape is calculated for a rectangular plate, and for a square plate the expression can be simplified drastically. To support the results, the model has been compared to a FEM model, and an excellent agreement between the two models is seen with a relative difference of less than $2 \%$ for all calculations. The model was also used to extract the cell capacitance, the parasitic capacitance and the residual stress of a pressure sensor composed of a multilayered plate of silicon and silicon oxide. The extracted values were in good agreement with the expected and it showed that the behavior of devices with a plate could easily be predicted with a low uncertainty.
\end{abstract}

Keywords: Anisotropic plate theory, Micromechanics, Stress, Multilayers

\section{Introduction}

Thin-walled structures such as plates are encountered in many branches of technology, from aircraft structures to actuators in microtechnology. If more than one layer is combined, advantages over homogeneous plates can be achieved, as for instance increased strength and lower weight. This is particularly desirable in aerospace technology where minimizing weight is of great importance. In microtechnology, plates can be used for pressure sensors [1-4] and Capacitive Ultrasonic Micromachined Transducers (CMUTs) [5-10], where it may not be desirable to make the devices of only one layer. For both applications, each layer may posses different thicknesses, orientation and anisotropic elastic properties and may have a residual stress. So to predict the performance of the device, it is needed to be able to predict the behavior of the multilayered plate.

The behavior of isotropic plates is well known and has been treated in detail by several authors [11-14]. A brief overview of the main results of the isotropic plate behavior is given here to be able to show afterwards that the developed model in this work can be simplified to these exact equations.

To determine the deformation and stresses in monolayered isotropic plates Kirchhoff-Love theory of plates is used, and the plate equation is [14]

$$
\begin{aligned}
& \frac{\partial w^{4}}{\partial x^{4}}+2 \frac{\partial^{4} w}{\partial x^{2} \partial y^{2}}+\frac{\partial w^{4}}{\partial y^{4}} \\
& =\frac{1}{D_{i}}\left(-q+N_{1} \frac{\partial w^{2}}{\partial x^{2}}+N_{2} \frac{\partial w^{2}}{\partial y^{2}}+2 N_{6} \frac{\partial w^{2}}{\partial x \partial y}\right)
\end{aligned}
$$

here $q$ is the pressure difference across the plate, $N$ is the stress resultant related by $\left(N_{1}, N_{2}, N_{6}\right)=\left(N_{x x}, N_{y y}, N_{x y}\right)$, and $D_{i}$ is the isotropic flexural rigidity, given by

$$
D_{i}=\frac{E}{12\left(1-v^{2}\right)} h^{3}
$$

where $E$ is Young's modulus, $v$ is Poisson's ratio, and $h$ is the thickness of the plate.

If the plate equation is solved using proper boundary conditions, the deflection surface can be used to calculate the stress and strain distributions. If we consider a circular plate without any stress resultant, $N=0$, the deflection mode, $w$, is described by [14]

$$
w(r)=w_{0}\left(1-\left(\frac{r}{a}\right)^{2}\right)^{2}
$$

where $a$ is the radius of the plate, $r$ is the radial coordinate and $w_{0}$ is the center deflection given by

$$
w_{0}=\frac{q a^{4}}{64 D_{i}} .
$$

If we instead neglect the stiffness of the plate, $D_{i}$, and assume uniform biaxial stress, it is called a membrane, in this case the deflection shape of a circular membrane can be described as [14]

$$
w(r)=w_{0}\left(1-\left(\frac{r}{a}\right)^{2}\right)
$$

where the center deflection, $w_{0}$, is 


$$
w_{0}=\frac{q a^{2}}{4 N} .
$$

If a plate both have a stiffness and a compressive residual stress, Timoshenko [13] has shown that the deflection shape is described by

$$
\begin{array}{r}
w(r)=\frac{q a}{2} \sqrt{\frac{D_{i}}{N^{3}}}\left(\frac{\mathrm{J}_{0}\left(\sqrt{\frac{N}{D_{i}}} r\right)-\mathrm{J}_{0}\left(\sqrt{\frac{N}{D_{i}}} a\right)}{\mathrm{J}_{1}\left(\sqrt{\frac{N}{D_{i}}} a\right)}\right) \\
-\frac{q a^{2}}{4 N}\left(1-\left(\frac{r}{a}\right)^{2}\right)
\end{array}
$$

where $\mathrm{J}_{n}$ is the Bessel function of first kind of order $n$.

In general, two different approaches have been used to study multilayered plates, equivalent layer theory and discrete layer theory [15]. In equivalent layer theory, every layer is assumed to behave as one equivalent layer, whereas in the discrete layer theory, every layer is considered individually. The equivalent layer theory can be divided into two subcategories, classical laminated plate theory (CLPT) and shear deformation theory (SDT) [15].

The most simple form of equivalent layer theory is the CLPT [16-20]. The theory is based on the work by Kirchoff [11], Love [12] and Timoshenko [13]. The theory is build on the assumption that the lines normal to the mid-surface remain normal and straight to the mid-surface after deformation [21], leading to the effect of transverse shear stress is ignored. This theory is therefore only valid for thin plates, but is less computational time consuming compared to other theories.

To analyze thicker plates, SDT must be used [22-25]. These are often based on the theories developed by Reissner [26] and Mindlin [27], which are an extension to the Kirchoff-Love theory which takes shear deformation through the thickness of the plate into account.

Discrete layer theories have been presented to achieve even more accurate results [28-31]. These theories has many unknowns for multilayered plates, and the number of unknowns increases with the number of layers. This makes the theory computational time consuming to obtain accurate results.

Pister et al. [16] formulated a system of equations governing the elastic bending of a plate consisting of two or more thin layers of isotropic material. The system was formulated to resemble the isotropic plate equation (1), and they showed that it reduced to the same equation for a single layer.

Thomsen et al. [32] formulated an analytic description of anisotropic single-layered plates made of silicon, and showed that errors of up to $10-25 \%$ in the center deflection was the result of using the isotropic description instead of the anisotropic description.

Dong et al. [18] developed a small-deflection theory governing the elastic bending of thin laminated anisotropic shells and plates composed of an arbitrary number of bonded layers. The moments and stress results are calculated from the stress-strain relation for each layer, and the from these they formulate a system of equations governing the behavior of laminated shells.
In this paper we extend the theory by Dong et al. [18] to achieve an analytical approach for modeling of shell-like plates with an arbitrary number of anisotropic layers with residual stress.

The structure of the paper is as follows: First, the derivation of the multilayered plate equation is covered build on the classical laminated plate theory, to develop a description of thin multilayered plates. The derivation of the bending moments and the stress resultant will follow the derivations from Dong et al. [18], and being a further development of their theory. Then an exact solution to circular plates is derived, and a expression for the buckling limit and the resonance frequency is derived. This is followed a description of rectangular plates based on a approximation method, and hereafter is it shown that this can be extremely simplified for square plates. Finally an example of the theory is given based on real devices.

\section{Materials and Methods}

The analytical calculation is carried out in Mathematica 10, and is compared to a finite element method (FEM) calculation performed in COMSOL 4.4 with a full stiffness tensor describing each material. A circular and square plate of (100) silicon and with isotropic aluminum on top was modeled with radius/side length of $100 \mu \mathrm{m}$ and a total plate thickness of $2 \mu \mathrm{m}$. This aspect ratio was chosen in order to neglect the effect of shear deformation [32]. The square plate was aligned to the [110] direction and the symmetry of the plates was utilized so only an eighth of the plate had to be simulated. The anisotropy was taken into account by using the appropriate $6 \times 6$ stiffness matrix and the stress in the layers are modeled in COMSOL as a bi-axial stress. The material parameters used in this paper are found in Table 1. The multilayered plate is meshed in FEM with a triangular mesh on the top surface and the mesh was repeated seven times through the plate, since the simulations were found to converge at this point. The mesh through the plate was set to adapt to the thickness of the different layers, so the mesh height was the same for each mesh layer, and independent of the material layer thickness. The Voight notation is used throughout this paper in the numbered coordinate system and the relation of the stress tensor notation to the Cartesian coordinate system is shown here

$$
\begin{aligned}
\sigma & =\left(\sigma_{1}, \sigma_{2}, \sigma_{3}, \sigma_{4}, \sigma_{5}, \sigma_{6}\right)^{\mathrm{T}} \\
& =\left(\sigma_{x x}, \sigma_{y y}, \sigma_{z z}, \sigma_{y z}, \sigma_{x z}, \sigma_{x y}\right)^{\mathrm{T}} .
\end{aligned}
$$

\section{Multilayered Plate equation}

We consider a thin plate composed of $n$ layers as shown in Figure 1, where each layer is anisotropic, and we assume plane stress. This assumption of plane stress is valid when a plate is thin compared to its lateral dimension, all the stresses perpendicular to the plate are then negligible compared to those parallel to it, i.e. $\sigma_{3}=\sigma_{4}=\sigma_{5}=0$. Thomsen et al. showed that 
Table 1: Material parameters of aluminum and silicon. The room temperature stiffness coefficient for high doped $\left(2.1 \times 10^{19} \mathrm{~cm}^{-3}\right)$ n-type crystalline silicon was measured by [33].

\begin{tabular}{l|ccc}
\hline \hline Parameter & $\begin{array}{c}\text { Aluminum } \\
\text { (Isotropic) }\end{array}$ & $\begin{array}{c}\mathrm{SiO}_{2} \\
\text { (Isotropic) }\end{array}$ & $\begin{array}{c}\text { Silicon } \\
\text { (Anisotropic) }\end{array}$ \\
\hline Young's modulus & $70 \mathrm{GPa}$ & $70 \mathrm{GPa}$ & - \\
Poisson's ratio & 0.35 & 0.17 & - \\
& - & - & $c_{11}=163.94 \mathrm{GPa}$ \\
Stiffness values & - & - & $c_{12}=64.77 \mathrm{GPa}$ \\
& - & - & $c_{44}=79.19 \mathrm{GPa}$ \\
Density & $2700 \mathrm{~kg} / \mathrm{m}^{3}$ & $2200 \mathrm{~kg} / \mathrm{m}^{3}$ & $2330 \mathrm{~kg} / \mathrm{m}^{3}$ \\
\hline \hline
\end{tabular}

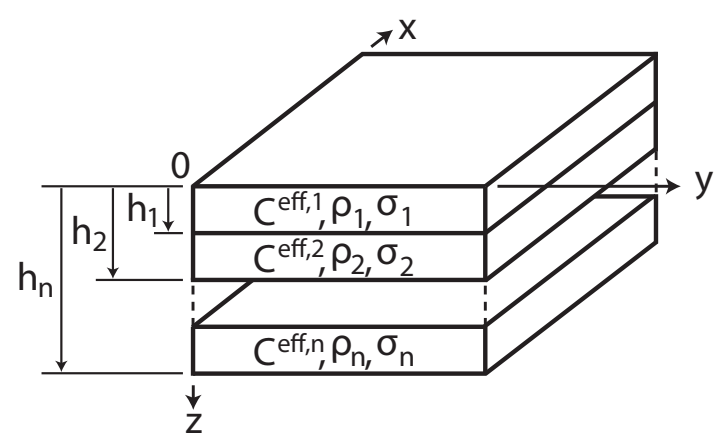

Figure 1: A sketch of a part of a plate with an arbitrary number of layers.

the error of the center deflection based on this assumption is no greater than $1 \%$ for aspect ratios greater than 20 [32]. Using the plane-stress condition the stiffness tensor can be reduced to a $3 \times 3$ matrix, and the stress-strain relation of the $k$ 'th layer can be written as

$$
\begin{aligned}
\left(\begin{array}{l}
\sigma_{1} \\
\sigma_{2} \\
\sigma_{6}
\end{array}\right)_{(k)}=\left(\begin{array}{lll}
S_{11} & S_{12} & S_{16} \\
S_{12} & S_{22} & S_{26} \\
S_{16} & S_{26} & S_{66}
\end{array}\right)_{(k)}^{-1}\left(\begin{array}{l}
\varepsilon_{1} \\
\varepsilon_{2} \\
\varepsilon_{6}
\end{array}\right) \\
=\left(\begin{array}{lll}
C_{11}^{\text {eff }} & C_{12}^{\text {eff }} & C_{13}^{\text {eff }} \\
C_{12}^{\text {eff }} & C_{22}^{\text {eff }} & C_{23}^{\text {eff }} \\
C_{13}^{\text {eff }} & C_{23}^{\text {eff }} & C_{33}^{\text {eff }}
\end{array}\right)_{(k)}\left(\begin{array}{l}
\varepsilon_{1} \\
\varepsilon_{2} \\
\varepsilon_{6}
\end{array}\right)
\end{aligned}
$$

here the effective stiffness matrix from the reduced compliance matrix is defined as $\mathbf{C}_{\mathrm{eff}}=\left(\mathbf{S}_{\mathrm{eff}}\right)^{-1}$.

The strains in the plate with the small deflection approximation can on kinematic grounds [34] be written as

$$
\left(\begin{array}{l}
\varepsilon_{1} \\
\varepsilon_{2} \\
\varepsilon_{6}
\end{array}\right)=\left(\begin{array}{l}
\varepsilon_{1,0} \\
\varepsilon_{2,0} \\
\varepsilon_{6,0}
\end{array}\right)-z\left(\begin{array}{c}
\frac{\partial^{2} w}{\partial x^{2}} \\
\frac{\partial^{2} w}{\partial y^{2}} \\
2 \frac{\partial^{2} w}{\partial x \partial y}
\end{array}\right)
$$

where $w$ is the deflection and $\left(\varepsilon_{1,0}, \varepsilon_{2,0}, \varepsilon_{6,0}\right)$ is the strain at the top of the plate at $z=0$.

The bending moments, $M$, and the stress resultants, $N$, can be found in terms of the top surface strain by substituting (10) into (9), integrating over each layer and summing over $n$ layers

$$
\begin{aligned}
& \left(\begin{array}{l}
M_{1} \\
M_{2} \\
M_{6}
\end{array}\right)=\sum_{k=1}^{n} \int_{h_{k-1}}^{h_{k}}\left(\begin{array}{l}
\sigma_{1} \\
\sigma_{2} \\
\sigma_{6}
\end{array}\right)_{(k)} z d z \\
& \left(\begin{array}{l}
N_{1} \\
N_{2} \\
N_{6}
\end{array}\right)=\sum_{k=1}^{n} \int_{h_{k-1}}^{h_{k}}\left(\begin{array}{l}
\sigma_{1} \\
\sigma_{2} \\
\sigma_{6}
\end{array}\right)_{(k)} d z .
\end{aligned}
$$

Performing the above integration yields

$$
\begin{aligned}
& \left(\begin{array}{l}
M_{1} \\
M_{2} \\
M_{6}
\end{array}\right)=\left(\begin{array}{lll}
b_{11} & b_{12} & b_{13} \\
b_{12} & b_{22} & b_{23} \\
b_{13} & b_{23} & b_{33}
\end{array}\right)\left(\begin{array}{l}
\varepsilon_{1,0} \\
\varepsilon_{2,0} \\
\varepsilon_{6,0}
\end{array}\right)-\left(\begin{array}{lll}
g_{11} & g_{12} & g_{13} \\
g_{12} & g_{22} & g_{23} \\
g_{13} & g_{23} & g_{33}
\end{array}\right)\left(\begin{array}{c}
\frac{\partial^{2} w}{\partial x^{2}} \\
\frac{\partial^{2} w}{\partial y^{2}} \\
2 \frac{\partial^{2} w}{\partial x \partial y}
\end{array}\right) \\
& \left(\begin{array}{l}
N_{1} \\
N_{2} \\
N_{6}
\end{array}\right)=\left(\begin{array}{lll}
a_{11} & a_{12} & a_{13} \\
a_{12} & a_{22} & a_{23} \\
a_{13} & a_{23} & a_{33}
\end{array}\right)\left(\begin{array}{l}
\varepsilon_{1,0} \\
\varepsilon_{2,0} \\
\varepsilon_{6,0}
\end{array}\right)-\left(\begin{array}{lll}
b_{11} & b_{12} & b_{13} \\
b_{12} & b_{22} & b_{23} \\
b_{13} & b_{23} & b_{33}
\end{array}\right)\left(\begin{array}{c}
\frac{\partial^{2} w}{\partial x^{2}} \\
\frac{\partial^{2} w}{\partial y^{2}} \\
2 \frac{\partial^{2} w}{\partial x \partial y}
\end{array}\right)
\end{aligned}
$$

where the constants $a_{i j}, b_{i j}$ and $g_{i j}$ are defined as

$$
\begin{aligned}
& a_{i j}=\sum_{k=1}^{n}\left(h_{k}-h_{k-1}\right) C_{i j}^{\mathrm{eff}, \mathrm{k}} \\
& b_{i j}=\frac{1}{2} \sum_{k=1}^{n}\left(h_{k}^{2}-h_{k-1}^{2}\right) C_{i j}^{\mathrm{eff}, \mathrm{k}} \\
& g_{i j}=\frac{1}{3} \sum_{k=1}^{n}\left(h_{k}^{3}-h_{k-1}^{3}\right) C_{i j}^{\mathrm{eff}, \mathrm{k}}
\end{aligned}
$$

and are matrix components in $\mathbf{A}, \mathbf{B}$ and $\mathbf{G}$. By combining equation (13) and (14), we can eliminate the surface strain, and the bending moments, can in abbreviated matrix notation, be written

$$
\mathbf{M}=\mathbf{F}[\mathbf{N}+\mathbf{B} \gamma]-\mathbf{G} \gamma
$$

where $\mathbf{F}=\mathbf{B} \mathbf{A}^{-1}$ and $\gamma=\left(\frac{\partial^{2} w}{\partial x^{2}}, \frac{\partial^{2} w}{\partial y^{2}}, 2 \frac{\partial^{2} w}{\partial x \partial y}\right)^{\mathrm{T}}$. By considering the bending moment and stress resultant balances, the equation of equilibrium for a plate is [16]

$$
\begin{aligned}
\frac{\partial^{2} M_{1}}{\partial x^{2}}+2 \frac{\partial^{2} M_{6}}{\partial x \partial x}+\frac{\partial^{2} M_{2}}{\partial y^{2}} & \\
= & \left(-q+N_{1} \frac{\partial^{2} w}{\partial x^{2}}+2 N_{6} \frac{\partial^{2} w}{\partial x \partial y}+N_{2} \frac{\partial^{2} w}{\partial y^{2}}\right) .
\end{aligned}
$$

If we insert the bending moments, equation (18), into equation (19) we can write

$$
\begin{aligned}
& \frac{\partial^{4} w}{\partial x^{4}}+k_{1} \frac{\partial^{4} w}{\partial x^{3} \partial y}+k_{2} \frac{\partial^{4} w}{\partial x^{2} \partial y^{2}}+k_{3} \frac{\partial^{4} w}{\partial x \partial y^{3}}+k_{4} \frac{\partial^{4} w}{\partial y^{4}} \\
&=\frac{1}{D_{a}}\left(-q+N_{1} \frac{\partial^{2} w}{\partial x^{2}}+2 N_{6} \frac{\partial^{2} w}{\partial x \partial y}+N_{2} \frac{\partial^{2} w}{\partial y^{2}}\right)
\end{aligned}
$$


and we call this the generalized multilayered plate equation. The flexural rigidity, $D_{a}$, and the plate equation constants, $k_{n}$, are listed in Table 2.

\subsection{Two Layers}

We now investigate the general case of a plate having two layers of anisotropic material. The total thickness of the plate is $h=h_{2}$ and the thickness of the top plate is $h_{1}$. We will define the thickness ratio, $\alpha$, as the thickness of the top layer to the total plate thickness

$$
\alpha=\frac{h_{1}}{h} .
$$

To simplify the expressions, we will make a Taylor expansion at $\alpha=0$. This corresponds to the often encountered case where the plate has a thick base material and a thin layer on top. The flexural rigidity, $D_{a}$, and the plate equation constants, $k_{n}$, become

$$
\begin{aligned}
& D_{a}=\frac{1}{12} h^{3} C_{11}^{\mathrm{eff}, 2}+\frac{1}{4} h^{3}\left(C_{11}^{\mathrm{eff}, 1}-C_{11}^{\mathrm{eff}, 2}\right) \alpha \\
& k_{1}=4 \frac{C_{13}^{\mathrm{eff}, 2}}{C_{11}^{\mathrm{eff}, 2}}+12 \frac{C_{13}^{\mathrm{eff}, 1} C_{11}^{\mathrm{eff}, 2}-C_{11}^{\mathrm{eff}, 1} C_{13}^{\mathrm{eff}, 2}}{\left(C_{11}^{\mathrm{eff}, 2}\right)^{2}} \alpha \\
& k_{2}=2 \frac{C_{12}^{\mathrm{eff}, 2}+C_{33}^{\mathrm{eff}, 2}}{C_{11}^{\mathrm{eff}, 2}} \\
& +6 \frac{C_{11}^{\mathrm{eff}, 2}\left(C_{12}^{\mathrm{eff}, 1}+2 C_{33}^{\mathrm{eff}, 1}\right)-C_{11}^{\mathrm{eff}, 1}\left(C_{12}^{\mathrm{eff}, 2}+2 C_{33}^{\mathrm{eff}, 2}\right)}{\left(C_{11}^{\mathrm{eff}, 2}\right)^{2}} \alpha \\
& k_{3}=4 \frac{C_{23}^{\mathrm{eff}, 2}}{C_{11}^{\mathrm{eff}, 2}}+12 \frac{C_{23}^{\mathrm{eff}, 1} C_{11}^{\mathrm{eff}, 2}-C_{11}^{\mathrm{eff}, 1} C_{23}^{\mathrm{eff}, 2}}{\left(C_{11}^{\mathrm{eff}, 2}\right)^{2}} \alpha \\
& k_{4}=\frac{C_{22}^{\mathrm{eff}, 2}}{C_{11}^{\mathrm{eff}, 2}}+3 \frac{C_{22}^{\mathrm{eff}, 1} C_{11}^{\mathrm{eff}, 2}-C_{11}^{\mathrm{eff}, 1} C_{22}^{\mathrm{eff}, 2}}{\left(C_{11}^{\mathrm{eff}, 2}\right)^{2}} \alpha .
\end{aligned}
$$

For a single layer anisotropic plate, $\alpha=0$, it should be noted that the exact same expressions for the flexural rigidity and the plate equation constants are found as Thomsen et al. [32].

\section{Circular plates}

For a circular plate an exact solution to the plate equation (20) can be found. Due to the symmetry of a circular plate, we have that $\frac{\partial^{4} w}{\partial x^{3} \partial y}=\frac{\partial^{4} w}{\partial x \partial y^{3}}=0$. We assume that the residual stress in the layers is uniform biaxial stress, meaning that the same amount of stress is present in the two axial directions, which is a valid assumption for thin layers. We therefore have that $N_{1}=N_{2}=N$ and $N_{6}=0$. By utilizing the symmetry of a circular plate and the uniform biaxial residual stress, we can write the multilayered plate equation on a simple form

$$
\nabla^{4} w-\frac{N}{C_{d} D_{a}} \nabla^{2} w=-\frac{q}{C_{d} D_{a}}
$$

where

$$
C_{d}=\frac{3+k_{2}+3 k_{4}}{8}
$$

and the stress resultant is calculated as

$$
N=\sum_{k=1}^{n} \int_{h_{k-1}}^{h_{k}} \sigma_{0, k} d z
$$

where $\sigma_{0, k}$ is the residual stress in the k'th layer. However, it should be noted that $N$ also can describe the thermal stress in the plate, but this effect is not discussed in this paper. If we consider a clamped plate, the multilayered plate equation has two solutions depending on the sign of the stress resultant. The plate is said to be in a compressive state if the stress resultant is negative, $N=-\left|N_{c}\right|$, and in a tensile state if the stress resultant is positive, $N=\left|N_{t}\right|$. The compressive and the tensile solution are denoted $w_{c}$ and $w_{t}$, respectively

$$
\begin{array}{r}
w_{c}(r)=\frac{q a}{2} \sqrt{\frac{C_{d} D_{a}}{N_{c}^{3}}}\left(\frac{\mathrm{J}_{0}\left(\sqrt{\frac{N_{c}}{C_{d} D_{a}}} r\right)-\mathrm{J}_{0}\left(\sqrt{\frac{N_{c}}{C_{d} D_{a}}} a\right)}{\mathrm{J}_{1}\left(\sqrt{\frac{N_{c}}{C_{d} D_{a}}} a\right)}\right) \\
-\frac{q a^{2}}{4 N_{c}}\left(1-\left(\frac{r}{a}\right)^{2}\right) \\
w_{t}(r)=-\frac{q a}{2} \sqrt{\frac{C_{d} D_{a}}{N_{t}^{3}}}\left(\frac{\mathrm{I}_{0}\left(\sqrt{\frac{N_{t}}{C_{d} D_{a}}} r\right)-\mathrm{I}_{0}\left(\sqrt{\frac{N_{t}}{C_{d} D_{a}}} a\right)}{\mathrm{I}_{1}\left(\sqrt{\frac{N_{t}}{C_{d} D_{a}}} a\right)}\right)^{+\frac{q a^{2}}{4 N_{t}}}\left(1-\left(\frac{r}{a}\right)^{2}\right)
\end{array}
$$

where $\mathrm{J}_{n}(x)$ is the Bessel function of first kind and $\mathrm{I}_{n}(x)$ is the modified Bessel function of first kind. The center deflection is found at $r=0$ and can be written

$$
\begin{gathered}
w_{0, c}=\frac{q a}{2} \sqrt{\frac{C_{d} D_{a}}{N_{c}^{3}}}\left(\frac{1-\mathrm{J}_{0}\left(\sqrt{\frac{N_{c}}{C_{d} D_{a}}} a\right)}{\mathrm{J}_{1}\left(\sqrt{\frac{N_{c}}{C_{d} D_{a}}} a\right)}\right)-\frac{q a^{2}}{4 N_{c}} \\
w_{0, t}=-\frac{q a}{2} \sqrt{\frac{C_{d} D_{a}}{N_{t}^{3}}}\left(\frac{1-\mathrm{I}_{0}\left(\sqrt{\frac{N_{t}}{C_{d} D_{a}}} a\right)}{\mathrm{I}_{1}\left(\sqrt{\frac{N_{t}}{C_{d} D_{a}}} a\right)}\right)+\frac{q a^{2}}{4 N_{t}} .
\end{gathered}
$$

For a single layer plate of isotropic material this solution reduces to the already known plate equation, equation (7), as one would expect.

We can investigate how the solution behaves when stiffness is ignored and when residual stress is ignored. When stiffness is ignored, we find the limit for $D_{a} \rightarrow 0$

$$
\lim _{D_{a} \rightarrow 0} w=\frac{q a^{2}}{4 N}\left(1-\left(\frac{r}{a}\right)^{2}\right) .
$$

This is the solution to bending of a membrane, equation (5), as one would expect. 
Table 2: The plate equation coefficients from equation (20), $D_{a}$ is the flexural rigidity and $k_{n}$ are the plate equation constants.

\begin{tabular}{|l|}
\hline Parameter \\
\hline$D_{a}=g_{11}-b_{11} c_{11}+b_{12} f_{12}+b_{13} f_{13}$ \\
\hline$k_{1} D_{a}=2\left(b_{23} f_{12}+\left(b_{11}+b_{33}\right) f_{13}+b_{12} f_{23}+b_{13}\left(f_{11}+f_{33}\right)-2 g_{13}\right)$ \\
\hline$k_{2} D_{a}=\left(b_{11}+b_{22}\right) f_{12}+b_{12}\left(f_{11}+f_{22}\right)+b_{13}\left(4 f_{13}+f_{23}\right)+b_{23}\left(f_{13}+4 f_{23}\right)+4 b_{33} f_{33}-2 g_{12}-4 g_{33}$ \\
\hline$k_{3} D_{a}=2\left(b_{13} f_{12}+b_{12} f_{13}+\left(b_{22}+b_{33}\right) f_{23}+b_{23}\left(f_{22}+f_{33}\right)-2 g_{23}\right)$ \\
\hline$k_{4} D_{a}=b_{12} f_{12}+b_{22} f_{22}+b_{23} f_{23}-g_{22}$ \\
\hline
\end{tabular}

When residual stress is ignored, we find the limit for $N \rightarrow 0$

$$
\lim _{N \rightarrow 0} w=\frac{q a^{4}}{64 C_{d} D_{a}}\left(1-\left(\frac{r}{a}\right)^{2}\right)^{2}
$$

and is the solution to bending of an anisotropic plate with zero residual stress as Thomsen et al. [32] showed. For an isotropic plate the deflection reduces to the known deflection shape, equation (3).

\subsection{Normalized deflection}

The deflection can be normalized to the center deflection of a plate without residual stress. If we now define two new variables, one for the compressive state, $\chi_{c}$, and one for the tensile state, $\chi_{t}$, which are defined as

$$
\chi_{c}=\sqrt{\frac{N_{c} a^{2}}{C_{d} D_{a}}}, \quad \chi_{t}=\sqrt{\frac{N_{t} a^{2}}{C_{d} D_{a}}}
$$

we can write the normalized deflections as

$$
\begin{gathered}
\frac{w_{c}}{w_{0}}=32 \frac{1}{\chi_{c}^{3}}\left(\frac{\mathrm{J}_{0}\left(\chi_{c} \frac{r}{a}\right)-\mathrm{J}_{0}\left(\chi_{c}\right)}{\mathrm{J}_{1}\left(\chi_{c}\right)}\right)-16 \frac{1}{\chi_{c}^{2}}\left(1-\left(\frac{r}{a}\right)^{2}\right) \\
\frac{w_{t}}{w_{0}}=-32 \frac{1}{\chi_{t}^{3}}\left(\frac{\mathrm{I}_{0}\left(\chi_{t} \frac{r}{a}\right)-\mathrm{I}_{0}\left(\chi_{t}\right)}{\mathrm{I}_{1}\left(\chi_{t}\right)}\right)+16 \frac{1}{\chi_{t}^{2}}\left(1-\left(\frac{r}{a}\right)^{2}\right) .
\end{gathered}
$$

The normalized deflection profiles then becomes a function of only the constant parameter $\chi$ and the relative position on the plate, $r / a$. Figure 2 shows the center deflection of a circular plate normalized to the center deflection at $N=0$. The data points represent the FEM calculations. Negative values of $\chi$ correspond to center deflections in the compressive state, $\chi_{c}$, and positives values correspond to center deflections in the tensile state, $\chi_{t}$. An excellent agreement between the analytical and the FEM calculations is seen for both the compressive and the tensile state, and with a relative difference of less than $1.6 \%$.

\subsection{Buckling}

In the compressive state, when the stress resultant becomes too big, buckling will occur. By examining the normalized deflection shape in the compressive state, equation (37), we see that the the first buckling mode will occur at the first root of the Bessel function of first kind of first order

$$
\mathrm{J}_{1}\left(\chi_{c}\right)=0
$$

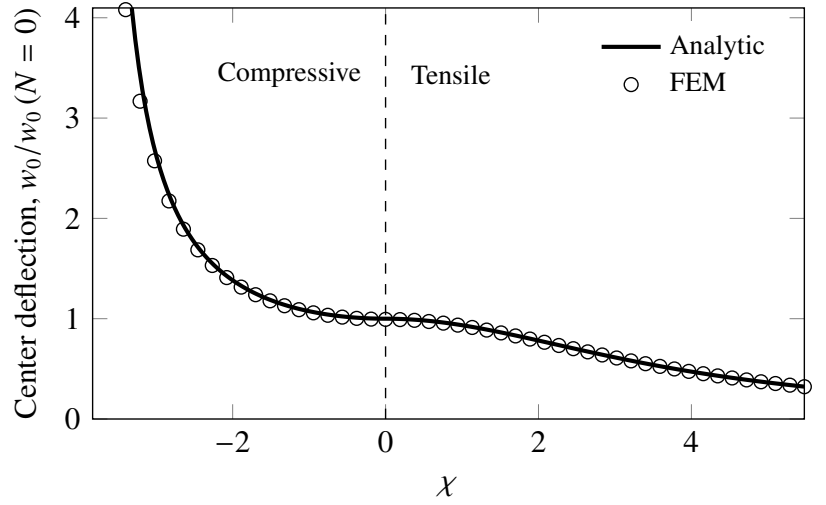

Figure 2: Center deflection normalized to the center deflection at $N=0$ plotted as a function of $\chi$ from (37) and (38). Negative $\chi$ corresponds to the compressive state $\left(\chi_{c}\right)$, and positive $\chi$ to the tensile state $\left(\chi_{t}\right)$.

The first root is found at $\chi_{c}=3.8317$ [35] and the critical stress resultant, $N_{c r i t}$, is then found as

$$
N_{c r i t}=-(3.8317)^{2} \frac{C_{d} D_{a}}{a^{2}}
$$

In the special case where we have the same amount of stress in all layers, we have that $N_{\text {crit }}=h \sigma_{\text {crit }}$ where $h$ is the total thickness of the plate and $\sigma_{c r i t}$ is the critical buckling stress. The flexural rigidity of a plate is proportional to $h^{3}$, as seen from equation (22), hence we can write the critical stress as

$$
\sigma_{c r i t}=-(3.8317)^{2} \frac{C_{d} D_{a}}{h^{3}}\left(\frac{h}{a}\right)^{2} .
$$

Figure 3 shows the critical stress for a single layer plate expressed as $\sigma_{c r i t} h^{3} /\left(C_{d} D_{a}\right)$. The data points represent the results from the FEM calculation, where the critical stress is computed from an eigenvalue problem. A linear relation between the critical stress and the inverse of the aspect ratio squared is seen as predicted from the analytical expression, equation (41). An excellent agreement between the two calculations is seen with a relative difference of less than $1.7 \%$ for aspect ratios greater than 10 .

\subsection{Natural Frequency}

The natural frequency of the plate can be estimated using the Rayleigh-Ritz method [14]

$$
\omega^{2}=\frac{\int_{A} w\left(C_{d} D_{a} \nabla^{4} w-N \nabla^{2} w\right) \mathrm{d} A}{\sum_{k=1}^{n} \int_{A} w\left(h_{k}-h_{k-1}\right) \rho_{k} w \mathrm{~d} A}
$$




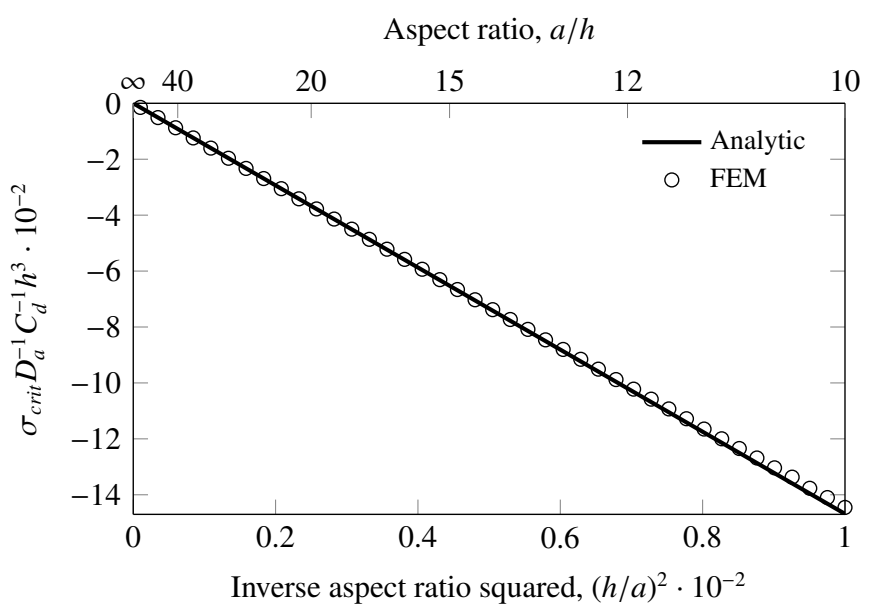

Figure 3: The critical limit for buckling plotted as function of the inverse aspect ratio squared in the case where we have the same stress in all layers. As predicted by equation (41) a linear dependence is seen for the FEM calculation.

where $\rho_{k}$ is the density of the k'th layer.

One of the strengths of the Rayleigh-Ritz method is that an approximate deflection shape results in an accurate result. Due to this, the deflection shape will be approximated with the shape of the plate without residual stress.

$$
\omega^{2}=\frac{20\left(16 C_{d} D_{a}+N a^{2}\right)}{3 a^{4} \sum_{k=1}^{n}\left(h_{k}-h_{k-1}\right) \rho_{k}} .
$$

The estimated natural frequency is always higher than the exact value, since the plate has been arbitrarily stiffened by the assumption of a modal shape, increasing the natural frequency.

Figure 4 shows the natural frequency normalized to the natural frequency with a zero stress resultant, $N=0$. The normalized natural frequency is reduced to

$$
\frac{\omega^{2}}{\omega_{0}^{2}}=1+\frac{1}{16} \frac{N a^{2}}{C_{d} D_{a}} .
$$

A linear relation between the stress resultant and the normalized natural frequency is seen, which is in excellent agreement with the FEM calculations, with relative differences between the calculations of less than $2 \%$.

It is noticeable that the FEM calculation predicts a slightly higher natural frequency than the Rayleigh-Ritz method, even though the Rayleigh-Ritz method yields a higher result than the exact value. This could be due to the difference of the clamped boundary condition. The analytical deflection shape must satisfy zero deflection and slope at the edge, where the FEM calculation is physically clamped. This makes the FEM model more stiff, since it can not contract or expand in the vertical direction at the edge.

\section{Rectangular Plates}

In contrast to circular plates, an exact solution to rectangular plates does not exist. Instead an approximate method has

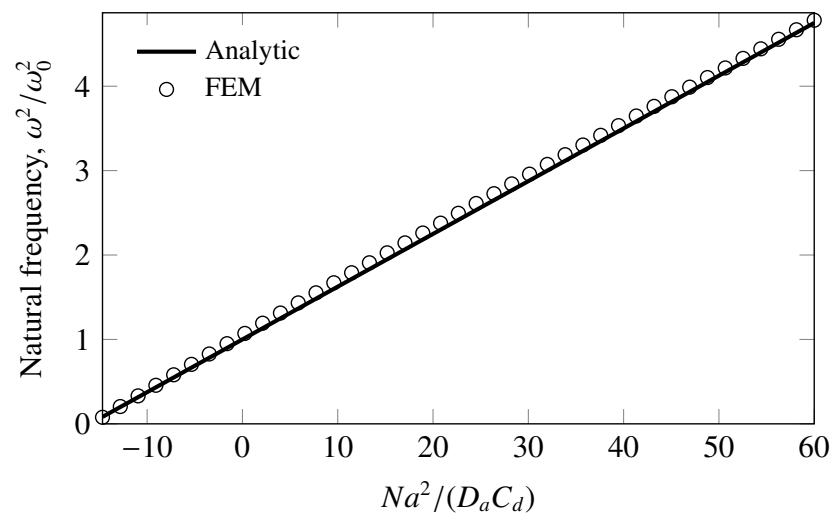

Figure 4: The natural frequency normalized to the natural frequency with a zero stress resultant. A linear relation between the stress resultant and the normalized natural frequency are seen as expected from (44).

to be used in order to calculate the deflection. The Galerkin method [14] is used to find approximate analytical deflections of rectangular and square plates.

Based on the generalized multilayered plate equation (20) we define the operator $\mathbf{H}$ as

$$
\begin{aligned}
\mathbf{H}=\frac{\partial^{4} w}{\partial x^{4}}+k_{1} \frac{\partial^{4} w}{\partial x^{3} \partial y}+ & k_{2} \frac{\partial^{4} w}{\partial x^{2} \partial y^{2}}+k_{3} \frac{\partial^{4} w}{\partial x \partial y^{3}} \\
+ & k_{4} \frac{\partial^{4} w}{\partial y^{4}}-\frac{N}{D_{a}}\left(\frac{\partial^{2} w}{\partial x^{2}}+\frac{\partial^{2} w}{\partial y^{2}}\right)
\end{aligned}
$$

such that the plate equation can be written

$$
\mathbf{H}+\frac{q}{D_{a}}=0
$$

where the deflection can be approximated by the following series

$$
w(x, y)=\sum_{k=0}^{k_{m}} \sum_{l=0}^{l_{m}} \lambda_{k l} \phi_{k l}
$$

where $\lambda_{k l}$ are the unknown coefficients which are to be determined, $\phi_{k l}$ is the linearly independent coordinate functions which satisfy the boundary conditions, and $k_{m}=k_{l}$ is the maximum summation index. The Galerkin system of equations $[32,36]$ is build as

$$
\begin{gathered}
\iint_{A} \phi_{00}\left(\mathbf{H}+\frac{p}{D_{a}}\right) \mathrm{d} x \mathrm{~d} y=0 \\
\iint_{A} \phi_{01}\left(\mathbf{H}+\frac{p}{D_{a}}\right) \mathrm{d} x \mathrm{~d} y=0 \\
\vdots \\
\iint_{A} \phi_{k_{m} l_{m}}\left(\mathbf{H}+\frac{p}{D_{a}}\right) \mathrm{d} x \mathrm{~d} y=0 .
\end{gathered}
$$

The deflection $w(x, y)$ is found by substituting (47) into (48) and solving the linear equation system to find the coefficients $\lambda_{k l}$. 
If we now consider a rectangular plate with sidelength $2 a$ and $2 b$ clamped along the edges, and we assume that

$$
\phi_{k l} \approx\left(x^{2}-a^{2}\right)^{2}\left(y^{2}-b^{2}\right)^{2} x^{k} y^{l}
$$

such that the trial function can be written as

$$
w \approx\left(x^{2}-a^{2}\right)^{2}\left(y^{2}-b^{2}\right)^{2} \sum_{k=0}^{k_{m}} \sum_{l=0}^{l_{m}} \lambda_{k l} x^{k} y^{l}
$$

we will have a trial function satisfying the clamped boundary condition of the rectangular plate and with a center deflection

$$
w_{0}=w(0,0)=\lambda_{00} a^{4} b^{4} .
$$

The number of terms in the trial function, can be selected from the expansion, equation (50). A higher number of terms results in a more precise approximation, but also complicates the calculations. We will consider the trial function

$$
\begin{aligned}
w^{o} & =\lambda_{00}^{o} \phi_{00}+\lambda_{11}^{o} \phi_{11}+\lambda_{20}^{o} \phi_{20}+\lambda_{02}^{o} \phi_{02} \\
& =\left(x^{2}-a^{2}\right)^{2}\left(y^{2}-b^{2}\right)^{2}\left(\lambda_{00}^{o}+\lambda_{11}^{o} x y+\lambda_{20}^{o} x^{2}+\lambda_{02}^{o} y^{2}\right)
\end{aligned}
$$

having four terms, with an uneven term in $x$ and $y$. If the trial function only consist of even terms in $x$ and $y$, it would lead to expressions only containing $k_{2}$ and $k_{4}$ and not $k_{1}$ and $k_{3}$. This is due to the terms in the plate equation containing $k_{1}$ and $k_{3}$ involves $\frac{\partial w^{4}}{\partial x^{3} \partial y}$ and $\frac{\partial w^{4}}{\partial x \partial y^{3}}$. When these derivatives are applied to uneven functions they will vanish when doing the integrals in equation (48). Even trial functions will therefore only be useful when $k_{1}=k_{3}=0$. The four term trial function contains uneven terms of $x$ and $y$ and will therefore lead to expression containing $k_{1}$ and $k_{3}$ which make it applicable on all types of plates. The parameters of the Galerkin expression are to lengthy to express, but can easily be calculated with this procedure.

\subsection{Natural Frequency}

To approximate the natural frequency of a rectangular plate, the Rayleigh-Ritz method can be used [14]. To estimate the natural frequency, the overall deflection shape is used as test function, equation (47), and the estimated natural frequency becomes

$$
\omega^{2}=\frac{9\left(7 b^{4}+2 a^{2} b^{2} k_{2}+7 a^{4} k_{4}\right) D_{a}+6 a^{2} b^{2}\left(a^{2}+b^{2}\right) N}{2 a^{4} b^{4} \sum_{k=1}^{n}\left(h_{k}-h_{k-1}\right) \rho_{k}} .
$$

\section{Square plates}

Due to the symmetry of square plates, the Galerkin expression can be simplified, and when the condition of plane stress is valid, only five different types of plates exist [32]. For type I, II and III the plate coefficients $k_{1}$ and $k_{3}$ are zero, and for type IV $k_{1}=-k_{3}$. For a square plate with sidelength $2 L$, we can neglect the terms containing $k_{1}$ and $k_{3}$ due to symmetry, and for plates of type I, II, III and IV we can write the generalized multilayered plate equation on the same simple form as for a circular plate, equation (27). Since $C_{d}$ is calculated from a transformation to polar coordinates, it will not be the same for square plates, since the transformation utilized the rotational symmetry in polar coordinates. If we compare the natural frequency of a circular plate, equation (43), to the natural frequency of a rectangular plate, equation (53), with $a=b$ we can identify an equivalent parameter to $C_{d}$, which we call $C_{s}$, containing all terms of $k_{2}$ and $k_{4}$, as

$$
C_{s}=\frac{7+2 k_{2}+7 k_{4}}{18} .
$$

We can now write the Galerkin operator $\mathbf{H}$ as

$$
\mathbf{H}=\frac{\partial^{4} w}{\partial x^{4}}+2 \frac{\partial^{4} w}{\partial x^{2} \partial y^{2}}+\frac{\partial^{4} w}{\partial y^{4}}-\frac{N}{C_{s} D_{a}}\left(\frac{\partial^{2} w}{\partial x^{2}}+\frac{\partial^{2} w}{\partial y^{2}}\right) .
$$

Since we do not have any terms containing $k_{1}$ and $k_{3}$ we can use a trial function without any uneven terms in $x$ and $y$. We will then consider the three term trial function for a square plate with side length $2 L$

$$
\begin{aligned}
w^{n} & =\lambda_{00}^{n} \phi_{00}+\lambda_{20}^{n} \phi_{20}+\lambda_{02}^{n} \phi_{02} \\
& =\left(x^{2}-L^{2}\right)^{2}\left(y^{2}-L^{2}\right)^{2}\left(\lambda_{00}^{n}+\lambda_{20}^{n} x^{2}+\lambda_{02}^{n} y^{2}\right)
\end{aligned}
$$

The calculated expressions of $\lambda_{00}^{n}, \lambda_{20}^{n}$ and $\lambda_{02}^{n}$ are

$$
\lambda_{00}^{n}=\frac{231 q\left(2421 C_{s} D_{a}+43 L^{2} N\right)}{256 L^{4}\left(9 C_{s} D_{a}\left(12015 C_{s} D_{a}+1154 L^{2} N\right)+163 L^{4} N^{2}\right)}
$$

$$
\begin{aligned}
\lambda_{20}^{n} & =\lambda_{02}^{n} \\
& =\frac{9009 q\left(36 C_{s} D_{a}+5 L^{2} N\right)}{512 L^{6}\left(9 C_{s} D_{a}\left(12015 C_{s} D_{a}+1154 L^{2} N\right)+163 L^{4} N^{2}\right)} .
\end{aligned}
$$

It is noted that $\lambda_{20}^{n}=\lambda_{02}^{n}$ due to the symmetry of a square plate. The center deflection $w_{0}^{n}$ is calculated as

$$
w_{0}^{n}=\lambda_{00}^{n} L^{8} \text {. }
$$

\subsection{Buckling}

The critical stress for buckling can be estimated by examining the center deflection. The center deflection must be finite, so when the denominator becomes zero buckling will occur. The equation that needs to be solved is a quadratic equation and will have two solutions. The first solution is the critical stress for the first buckling mode, and the second is an unphysical solution. The critical stress for buckling, $N_{\text {crit }}$, is calculated as

$$
N_{\text {crit }}=-13.1083 \frac{C_{s} D_{a}}{L^{2}} .
$$

It is noted that this buckling limit has the same form as for a circular plate, equation (40), just with a slightly different prefactor. As for for the circular plate, we can rewrite the buckling 


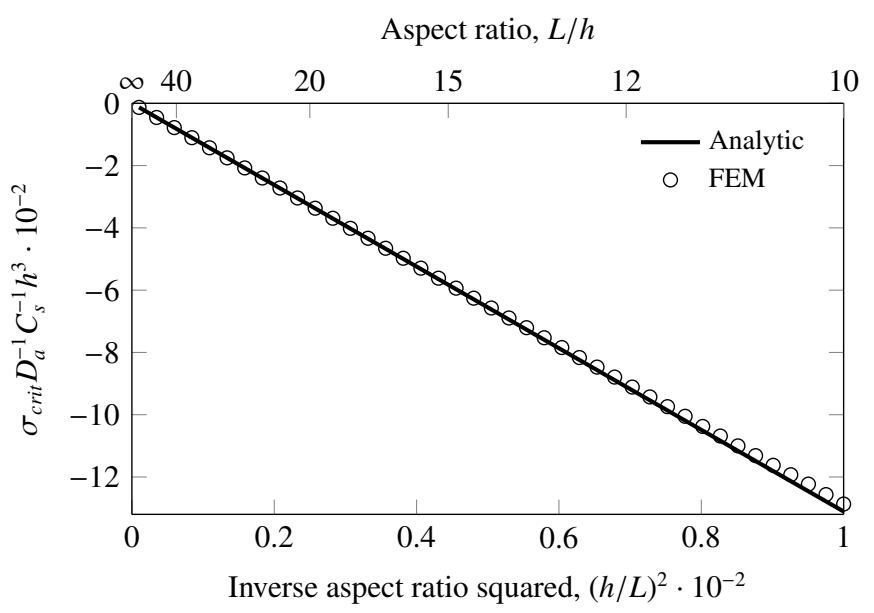

Figure 5: The critical limit for buckling plotted as function of the inverse aspect ratio squared in the case where we have the same stress in all layers. As predicted by (61) a linear dependence is seen for the FEM calculation.

limit in the special case where we have the same amount of stress in all layers to be inverse proportional to the aspect ratio squared

$$
\sigma_{\text {crit }}=-13.1083 \frac{C_{s} D_{a}}{h^{3}}\left(\frac{h}{L}\right)^{2} .
$$

Figure 5 shows the linear relation of the critical stress for a single layer plate expressed as $\sigma_{c r i t} h^{3} /\left(C_{d} D_{a}\right)$ as function of inverse of the aspect ratio squared. The data points represent the results from the FEM calculation, where the critical stress is computed from an eigenvalue problem, and the solid line represents the analytical calculation. An excellent agreement between the two calculations is seen with a relative difference of less than $2 \%$ for aspect ratios greater than 10 .

\subsection{Natural Frequency}

For a square plate the natural frequency can be calculated in the same way as for a rectangular plate, equation (53), by substituting $a=b=L$ and identifying the plate parameter $C_{s}$ we can write the natural frequency on this simple form

$$
\omega^{2}=\frac{81 C_{s} D_{a}+6 L^{2} N}{L^{4} \sum_{k=1}^{n}\left(h_{k}-h_{k-1}\right) \rho_{k}} .
$$

The natural frequency can be normalized to the natural frequency with a zero stress resultant, $N=0$, in the same way as the circular plate, equation (44). A linear relation between the normalized natural frequency squared and the stress resultant is seen as for the circular plate, but with a different slope

$$
\frac{\omega^{2}}{\omega_{0}^{2}}=1+\frac{2}{27} \frac{N L^{2}}{C_{s} D_{a}} .
$$

Figure 6 shows the normalized natural frequency squared as function of the stress resultant normalized to $D_{a} C_{s} / L^{2}$. An excellent agreement with the FEM calculations is seen, with relative differences between the two calculations of less than $2 \%$.

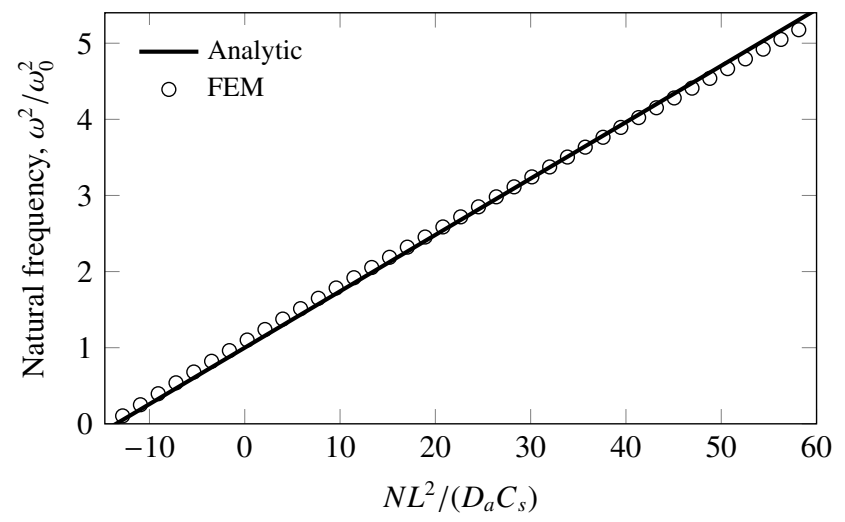

Figure 6: The natural frequency normalized to the natural frequency with a zero stress resultant. A linear relation between the stress resultant and the normalized natural frequency are seen also for a square plate.

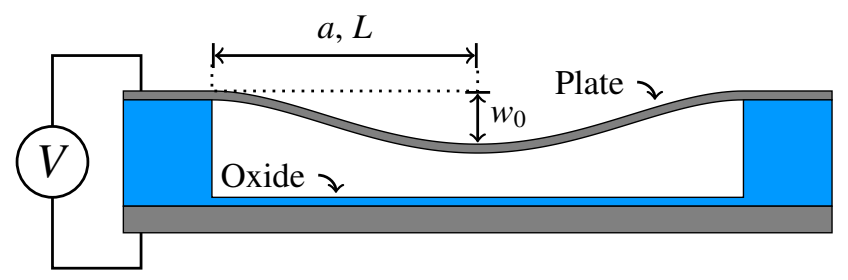

Figure 7: Cross sectional view of CMUT cell with applied voltage

\section{Example - CMUT}

A Capacitive Micromachined Ultrasonic Transducer (CMUT) [5-10], is one example where a plate is the essential part of the device. In its most simple form a CMUT can be understood as a plate capacitor, where the bottom electrode is fixed, and the top electrode, the plate, is free to move, as shown in Figure 7. The two electrodes are separated by an electrically insulating material clamping the plate at the edges, making it otherwise free to move. Typically CMUTs are fabricated using surface micromachining, where the device is built up by depositing and structuring multiple isotropic layers with residual stresses $[6,9]$. Another method is by wafer bonding, where the plate is typically anisotropic with an isotropic metal on top [8, 10]. This theory can be applied to both types of CMUTs, but in this example we will focus on a wafer bonding method where the plate is composed of two layers.

If we consider this thin double layered plate made of (100) silicon and with an isotropic aluminum layer on top, where the thickness ratio of the aluminum layer to the total plate thickness is defined as $\alpha$ as in equation (21). Depending on the conditions during deposition of the aluminum and its thermal history, the residual stress can vary from $-100 \mathrm{MPa}$ to above $300 \mathrm{MPa}$ [37]. To show the capabilities of the model different values of stress is modeled. The residual stress in the top aluminum layer is of different values from tensile to compressive ranging from $300 \mathrm{MPa}$ to $-80 \mathrm{MPa}$. To investigate the validity of the model analytical and FEM calculations were performed and compared. The comparison is made for both a circular and a square plate aligned to the [110] direction of the silicon crystal with the thickness ratios, $\alpha$, varying from 0 to 1 , with the 
end points corresponding to single layered plates of silicon and aluminum, respectively. For both calculations a total height of $2 \mu \mathrm{m}$ is used, the circular plate has a radius of $a=30 \mu \mathrm{m}$ and the square plate has a half width of $L=60 \mu \mathrm{m}$.

Figure 8 shows the center deflection normalized to a pure silicon plate $(\alpha=0)$. The calculations of a circular and a square plate are shown in (a) and (b), respectively. The FEM calculations are shown with marks and represent different residual stresses in the aluminum layer. In the same way, the normalized natural frequency is shown in Figure 9. When $\alpha$ is increased, meaning a bigger part of the plate is aluminum, the overall tendency is a softening of the plate, because aluminum is a softer material than silicon. This behavior is however also affected by the residual stress in the aluminum. A compressive stress will further soften the plate as one would expect, resulting in a higher center deflection or a lower natural frequency. Conversely, a tensile stress will stiffen the plate hence a lower center deflection or a higher natural frequency will be obtained. The analytical model is seen to predict the behavior of both multiple layers and take the residual stress into account, and is in good agreement with the FEM model with a relative difference of less than $2 \%$. The model presented here is clearly in very good agreement with FEM and efficient to use for design and modeling purposes.

The critical buckling stress limit can be calculated for all layer height ratios. The result is shown in Figure 10 for both a circular and square plate in (a) and (b), respectively. The area below the critical stress limit defines the stable regime where the plate will not experience buckling. Above the stress limit the plate will buckle, hence the behavior of the plate is not predictable. The square plate with an residual compressive stress of $80 \mathrm{MPa}$ is approaching the buckling regime in when the layer thickness ratio in approaching 1 . This can also be observed in Figure 8(b) where the center deflection increases dramatically compared to the other calculations.

\section{Example - Pressure Sensor}

Another example is the microfabricated pressure sensor. The capacitive pressure sensor is essentially the same device as the CMUT with a plate that deflects due the pressure difference across the plate. The change of capacitance when the plate is deflected can be detected and the pressure can be estimated. A pressure sensor with a plate of two layers fabricated using a wafer bonding technique was presented by Pedersen et al. [4]. The device consists of 180 hexagonal cells closely packed over large circular area and coupled in parallel. The plate is made of silicon with silicon oxide on top and the gap separating the top and bottom electrode is composed of vacuum and silicon oxide. The dimensional parameters of the pressure sensor are given in Table 3. The hexagonal cells can be approximated by circular cells, hence the center deflection of a circular plate with a compressive stress can be used to calculate the capacitance as function of pressure, equation (32). Using the center deflection $w_{0}$ the capacitance of each cell can be calculated as [38]
Table 3: Dimensional parameters of the pressure sensor.

\begin{tabular}{l|cc}
\hline \hline Parameter & Value & Unit \\
\hline Inscribed radius & 75 & $\mu \mathrm{m}$ \\
Number of cells, $n$ & 180 & - \\
Plate thickness, silicon & 2.30 & $\mu \mathrm{m}$ \\
Plate thickness, $\mathrm{SiO}_{2}$ & 576 & $\mathrm{~nm}$ \\
Effective gap, $g_{\text {eff }}$ & 426 & $\mathrm{~nm}$ \\
\hline \hline
\end{tabular}

$$
C_{\text {circ }}(p)=C_{0} \sqrt{\frac{g_{\text {eff }}}{w_{0}}} \operatorname{arctanh} \sqrt{\frac{w_{0}}{g_{\text {eff }}}}
$$

where $C_{0}$ is the capacitance of each cell at zero pressure, and $g_{\text {eff }}$ is the effective gap of the device [38]. The total capacitance of the device will however not only have a contribution from the cells, but also from the parasitics. Two parts are contributing to the parasitic capacitance, $C_{\text {para }}$, the bonding area between the cells and the measurement setup. The measured capacitance of the device then becomes

$$
C(p)=n C_{\text {circ }}(p)+C_{\text {para }} .
$$

The capacitance of the pressure sensor is measured with pressures varying from 250 to 1550 mbar. Using the dimensional and material parameters the behavior of the plate when a pressure is applied can be predicted, however the capacitance of the measurement system and the exact stress in the silicon oxide is unknown. The residual stress in the oxide depends on the process parameters, hence this parameter need to be fitted. These two parameters as well as the capacitance at zero pressure are extracted by fitting equation (65) to the measured data. The measurement and the fit are shown in Figure 11 together with the fitted and analytical calculated values. The black crosses represent the data and the solid line the fit. As seen in the figure the fitted parameters are consistent with the expected values. The deviance of $n C_{\text {circ }}(0)$ is only $1 \%$ and the stress in the oxide, $N$, is within the expected range of -200 to $-300 \mathrm{MPa}$. The parasitic capacitance is however higher than expected which is associated with the unknown parasitics of the packaging and the measurement setup. By establishing the amount of parasitics and the residual strss in the oxide, the behavior of multilayered pressure sensors could easily be predicted with a very low uncertainty, resulting in no calibration of the devices are needed.

\section{Conclusion}

In this paper we have presented an analytical approach for modeling of plates with an arbitrary number of anisotropic layers with residual stress. The multilayered plate equation has been developed from the bending moments and the stress resultants and by solving the plate equation the exact deflection profile has been calculated for circular plates. By using the Galerkin approximation the deflection profile of rectangular plates can be calculated, and it can be simplified for square plates. From the deflection profiles the buckling limits can be established. The natural frequency has been calculated using Rayleigh-Ritz method using the multilayered plate equation 


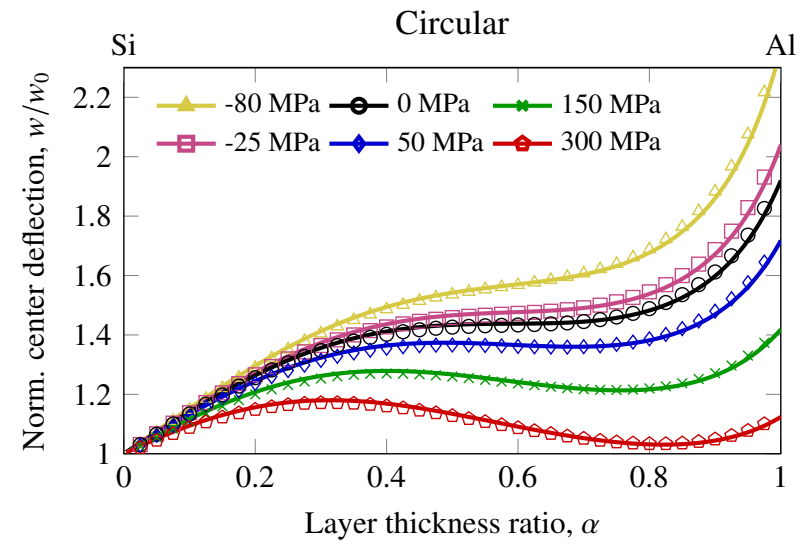

(a)

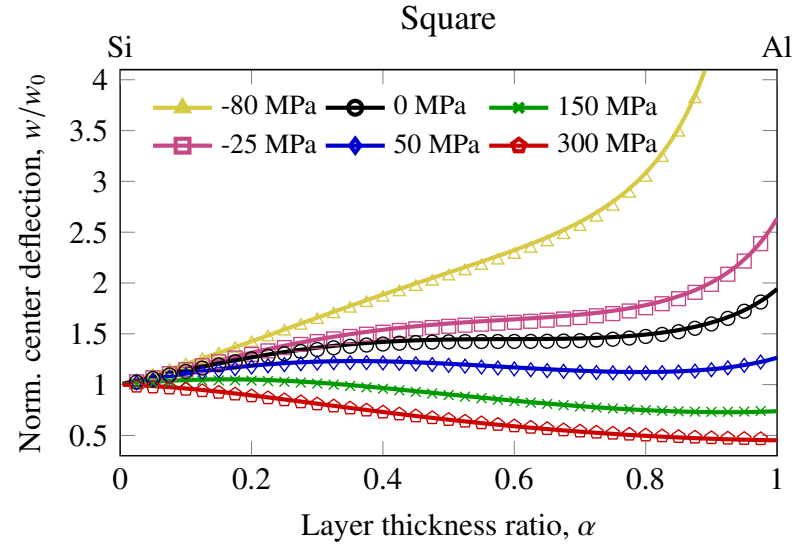

(b)

Figure 8: Center deflection of a multilayered plate of silicon and aluminum normalized to a pure silicon plate. The FEM calculations are shown with marks and represent different residual stresses in the aluminum layer. A circular plate is modeled in (a) and a square plate in (b). The maximum relative difference between the calculations are less than $1 \%$

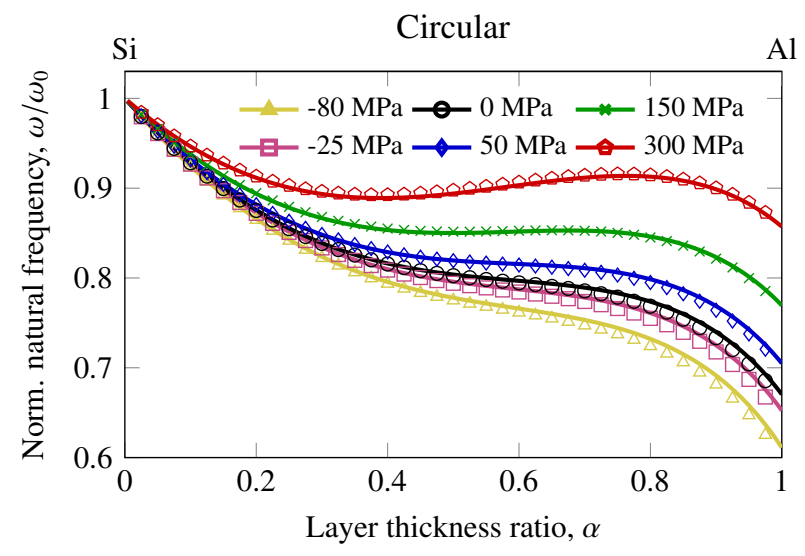

(a)

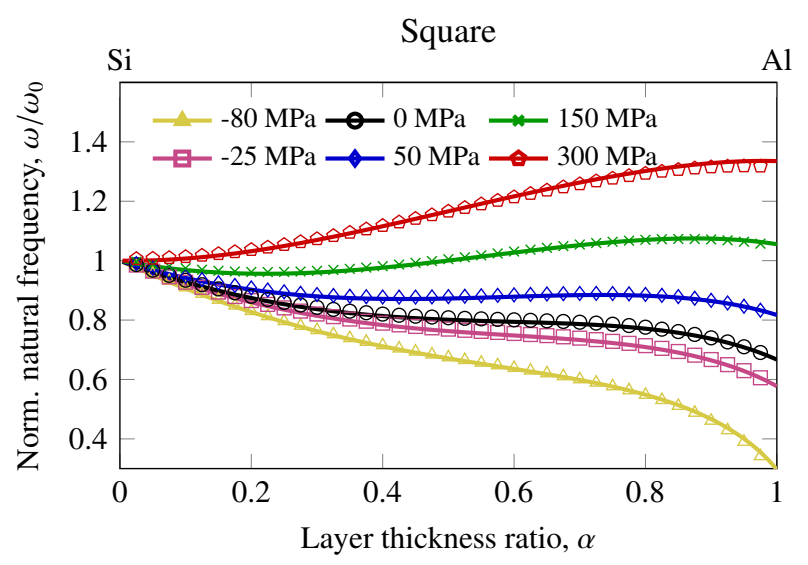

(b)

Figure 9: Natural frequency of a multilayered plate of silicon and aluminum normalized to a pure silicon plate. The FEM calculations are shown with marks and represent different residual stresses in the aluminum layer. A circular plate is modeled in (a) and a square plate in (b). The maximum relative difference between the calculations are less than $1.5 \%$

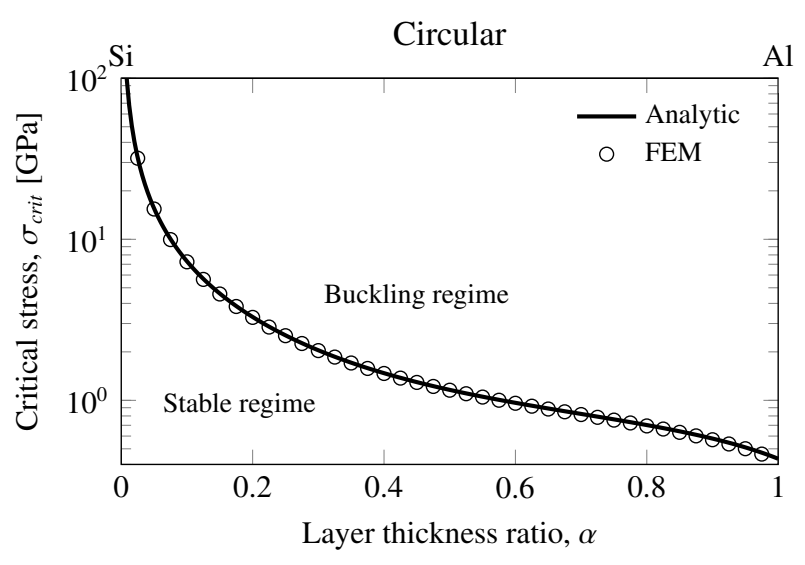

(a)

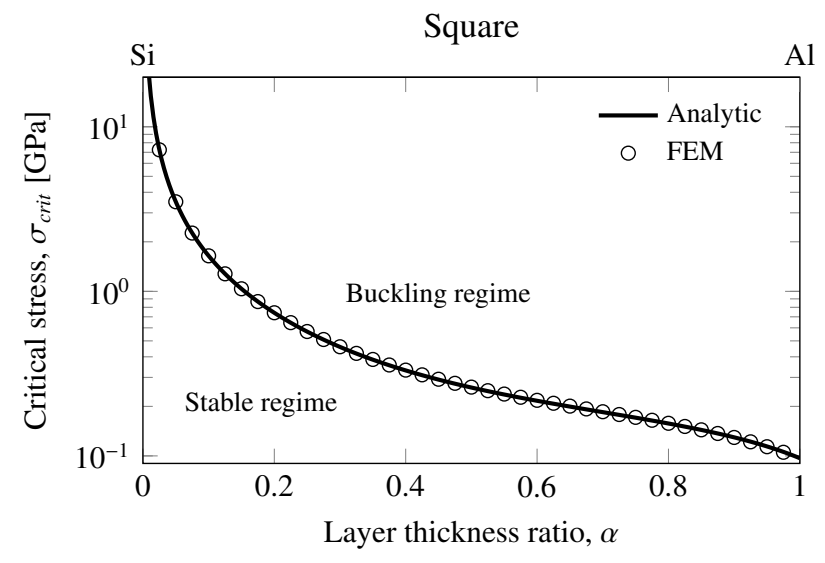

(b)

Figure 10: The critical stress for buckling of a multilayered plate of silicon and aluminum are calculated. Below the line, we have the stable regime where the plate will not experience buckling and above the plate will buckle. A circular plate is modeled in (a) and a square plate in (b). The maximum relative difference between the calculations are less than $1 \%$ 


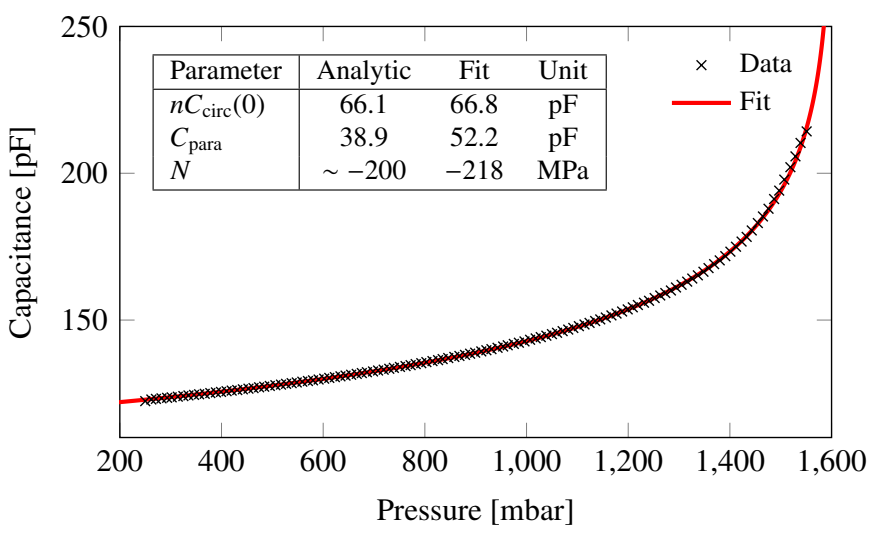

Figure 11: Measured capacitance as function of pressure based on a pressure sensor described in [4]. The crosses corresponds to the measured data and the solid line the fit. The fit parameters as well as the analytic calculated values are shown in the table.

and an approximate deflection profile. To verify the results, the analytical model has been compared to a FEM model, and an excellent agreement between the two models is seen with a relative difference of less than $2 \%$ for all calculations. As an example of the model a CMUT has been modeled, where the center deflection and the natural frequency is calculated for a doubled layered plate with varying height ratios and stress in one of the layers, for both circular and square plates. In the example, the model is compared to a FEM model, and an excellent agreement between the calculations are seen with a relative difference of less than $2 \%$. The model was also used to extract the cell capacitance, the parasitic capacitance and the residual stress of a pressure sensor composed of a multilayered plate of silicon and oxide. The extracted values were in good agreement with the expected and it showed that the behavior of a pressure sensor like this could easily be predicted with a low uncertainty. Besides being useful for analytical predictions of the behavior of plates, the model is very useful for tuning and optimization of FEM models since exact solutions to circular plates exist, and the Galerkin method results in a very precise estimate for rectangular plates.

\section{References}

[1] M. Habibi, E. Lueder, T. Kallfass, D. Horst, A surface micromachined capacitive absolute pressure sensor array on a glass substrate, Sensors and Actuators A: Physical 46 (1) (1995) 125-128.

[2] A. V. Chavan, K. D. Wise, Batch-processed vacuum-sealed capacitive pressure sensors, Microelectromechanical Systems, Journal of 10 (4) (2001) 580-588.

[3] P. Dimitropoulos, C. Kachris, D. Karampatzakis, G. Stamoulis, A new SOI monolithic capacitive sensor for absolute and differential pressure measurements, Sensors and Actuators A: Physical 123 (2005) 36-43.

[4] T. Pedersen, G. Fragiacomo, O. Hansen, E. V. Thomsen, Highly sensitive micromachined capacitive pressure sensor with reduced hysteresis and low parasitic capacitance, Sensors and Actuators A: Physical 154 (1) (2009) 35-41.

[5] D. W. Schindel, D. Hutchins, L. Zou, M. Sayer, et al., The design and characterization of micromachined air-coupled capacitance transducers, Ultrasonics, Ferroelectrics, and Frequency Control, IEEE Transactions on 42 (1) (1995) 42-50.
[6] M. Haller, B. T. Khuri-Yakub, et al., A surface micromachined electrostatic ultrasonic air transducer, Ultrasonics, Ferroelectrics, and Frequency Control, IEEE Transactions on 43 (1) (1996) 1-6.

[7] E. Cianci, V. Foglietti, G. Caliano, M. Pappalardo, Micromachined capacitive ultrasonic transducers fabricated using silicon on insulator wafers, Microelectronic engineering 61 (2002) 1025-1029.

[8] Y. Huang, E. Hæggström, M. H. Badi, B. Khuri-Yakub, et al., Fabricating capacitive micromachined ultrasonic transducers with wafer-bonding technology, Microelectromechanical Systems, Journal of 12 (2) (2003) 128-137.

[9] A. Caronti, G. Caliano, R. Carotenuto, A. Savoia, M. Pappalardo, E. Cianci, V. Foglietti, Capacitive micromachined ultrasonic transducer (CMUT) arrays for medical imaging, Microelectronics Journal 37 (8) (2006) 770-777.

[10] T. L. Christiansen, M. F. Rasmussen, J. P. Bagge, L. N. Møsner, J. Jensen, E. V. Thomsen, et al., 3-D imaging using row-column-addressed arrays with integrated apodization - part ii: transducer fabrication and experimental results, Ultrasonics, Ferroelectrics, and Frequency Control, IEEE Transactions on 62 (5) (2015) 959-971.

[11] G. Kirchhoff, Über das gleichgewicht und die bewegung einer elastischen scheibe., Journal für die reine und angewandte Mathematik 40 (1850) 5188.

[12] A. E. H. Love, The small free vibrations and deformation of a thin elastic shell, Philosophical Transactions of the Royal Society of London. A (1888) 491-546.

[13] S. Timoshenko, S. Woinowsky-Krieger, Theory of plates and shells, McGraw-Hill, 1959.

[14] E. Ventsel, T. Krauthammer, Thin plates and shells: theory, analysis, and applications, Dekker, 2001.

[15] M. Aydogdu, A new shear deformation theory for laminated composite plates, Composite Structures 89 (1) (2009) 94-101. doi:10.1016/j.compstruct.2008.07.008.

[16] K. S. Pister, S. B. Dong, A. M. ASCE, Elastic bending of layered plates, ASCE -Proceedings -Journal of the Engineering Mechanics Division 85 (1959) 1-10.

[17] E. Reissner, Y. Stavsky, Bending and stretching of certain types of heterogeneous aeolotropic elastic plates, Journal of Applied Mechanics 28 (3) (1961) 402-408.

[18] S. B. Dong, K. S. Pister, R. L. Taylor, On the theory of laminated anisotropic shells and plates, Journal of the aerospace sciences 29 (8) (1962) 969-975.

[19] R. D. Schile, Analysis of laminated circular plates, Journal of Composite Materials 1 (4) (1967) 324-335.

[20] G. H. Stickney, F. Abdulhadi, Flexure theory of multi-layer orthotropic circular sandwich plates, Journal of Composite Materials 2 (2) (1968) 200-219.

[21] J. N. Reddy, Theory and analysis of elastic plates and shells, CRC press, 2006.

[22] S. B. Dong, F. K. W. Tso, On a laminated orthotropic shell theory including transverse shear deformation, Journal of Applied Mechanics 39 (4) (1972) 1091-1097.

[23] J. M. Whitney, C. T. Sun, A higher order theory for extensional motion of laminated composites, Journal of Sound and Vibration 30 (1) (1973) 85-97.

[24] J. N. Reddy, C. F. Liu, A higher-order shear deformation theory of laminated elastic shells, International Journal of Engineering Science 23 (3) (1985) 319-330

[25] M. Di Sciuva, An improved shear-deformation theory for moderately thick multilayered anisotropic shells and plates, Journal of Applied Mechanics 54 (3) (1987) 589-596.

[26] E. Reissner, On bending of elastic plates, Quart. Appl. Math 5 (1) (1947) 55-68.

[27] R. D. Mindlin, Influence of rotary inertia and shear on flexural motions of isotropic, elastic plates, J. of Appl. Mech. 18 (1951) 31-38.

[28] K. N. Cho, C. W. Bert, A. G. Striz, Free vibrations of laminated rectangular plates analyzed by higher order individual-layer theory, Journal of sound and vibration 145 (3) (1991) 429-442.

[29] A. Nosier, R. K. Kapania, J. N. Reddy, Free vibration analysis of laminated plates using a layerwise theory, AIAA journal 31 (12) (1993) 23352346.

[30] D. H. Robbins, J. N. Reddy, Modelling of thick composites using a lay- 
erwise laminate theory, International Journal for Numerical Methods in Engineering 36 (4) (1993) 655-677.

[31] T. S. Plagianakos, D. A. Saravanos, Higher-order layerwise laminate theory for the prediction of interlaminar shear stresses in thick composite and sandwich composite plates, Composite Structures 87 (1) (2009) 23-35.

[32] E. V. Thomsen, K. Reck, G. Skands, C. Bertelsen, O. Hansen, Silicon as an anisotropic mechanical material: Deflection of thin crystalline plates, Sensors and Actuators A: Physical 220 (2014) 347-364.

[33] J. J. Hall, Electronic effects in the elastic constants of $n$-type silicon, Phys. Rev. 161 (1967) 756-761. doi:10.1103/PhysRev.161.756.

URL http://link . aps .org/doi/10.1103/PhysRev.161.756

[34] L. D. Landau, E. M. Lifshitz, Course of Theoretical Physics Vol 7: Theory and Elasticity, Butterworth-Heinemann, 1986.

[35] M. Abramowitz, I. A. Stegun, Handbook of mathematical funktions, Dover, 1965

[36] F. C. Mbakogu, M. N. Pavlovic, Bending of clamped orthotropic rectangular plates: a variational symbolic solution, Computers and Structures 77 (2) (2000) 117-128.

[37] P. A. Flinn, D. S. Gardner, W. D. Nix, Measurement and interpretation of stress in aluminum-based metallization as a function of thermal history, Electron Devices, IEEE Transactions on 34 (3) (1987) 689-699.

[38] M. F. la Cour, T. L. Christiansen, J. A. Jensen, E. V. Thomsen, Electrostatic and small-signal analysis of CMUTs with circular and square anisotropic plates, Ultrasonics, Ferroelectrics, and Frequency Control, IEEE Transactions on 62 (8) (2015) 1563-1579. 